\title{
Pembrolizumab Versus Combined Chemotherapy With Gemcitabine and Paclitaxel: A Comparative Assessment of Clinical Outcomes in Patients With Platinum-refractory Advanced Urothelial Cancer
}

\author{
AYANA TAKEMURA ${ }^{1,2}$, YUTO MATSUSHITA ${ }^{1}$, KEITA TAMURA $^{1}$, TAKAYUKI SUGIYAMA ${ }^{1}$, \\ MASAO NAGATA ${ }^{2}$, ATSUSHI OTSUKA ${ }^{1}$ and HIDEAKI MIYAKE ${ }^{1}$ \\ ${ }^{1}$ Department of Urology, Hamamatsu University School of Medicine, Hamamatsu, Japan; \\ ${ }^{2}$ Department of Urology, Hamamatsu Medical Center, Hamamatsu, Japan
}

\begin{abstract}
Background/Aim: There are limited data on comprehensive assessments of several treatments as second-line therapy against advanced urothelial cancer (UC). The objective of this study was to compare clinical outcomes between advanced UC patients receiving either pembrolizumab (Pem) or combined chemotherapy with gemcitabine and paclitaxel (GP) as second-line therapy. Patients and Methods: This study retrospectively analyzed the clinical outcomes of 89 patients with platinumrefractory advanced UC, consisting of 46 and 43 who received Pem and GP therapy, respectively, as second-line treatment. Results: There were no significant differences in major clinicopathological parameters between Pem and GP groups. No significant difference in the objective response rate was noted between the two groups. Progression-free survival (PFS) in the Pem group was significantly longer than that in the GP group; however, there was no significant difference in overall survival (OS) between them. Multivariate analyses identified performance status $\leq 2$ and liver metastasis as independent factors associated with poor outcomes in both PFS and OS. The incidence of adverse events in the GP group was significantly higher than that in the Pem group. Conclusion: Pem could be regarded as standard agent for platinum-refractory advanced UC patients.
\end{abstract}

This article is freely accessible online.

Correspondence to: Hideaki Miyake, Department of Urology, Hamamatsu University School of Medicine, 1-20-1 Handayama, Higashi-Ku, Hamamatsu 431-3192, Japan. Tel: +81 534352306, Fax: +81 534352305, e-mail: hideakimiyake@hotmail.com

Key Words: Advanced urothelial cancer, pembrolizumab, chemotherapy, second-line therapy.
Urothelial cancer (UC) of the urinary tract represents the fourth most common type of malignancy worldwide (1). Approximately 30 and 5\% of UC patients present with muscle invasive and metastatic diseases, respectively, at initial diagnosis. Furthermore, despite adequate local treatment of patients with muscle invasive UC by radical surgery, metastatic disease progression ultimately occurs in more than one-third of these patients (2). To date, systemic chemotherapy using a platinum-based regimen, particularly combined chemotherapy with gemcitabine and cisplatin, has been the gold-standard treatment for advanced UC patients as first-line therapy (3); however, no second-line treatment has been established, and the prognosis of advanced UC patients following failure of first-line chemotherapy has been reported to be extremely poor with an overall survival period of $<1$ year (4).

The recent introduction of immune checkpoint inhibitors (ICIs), targeting major molecules mediating immune checkpoint pathways, such as programmed death (PD)-1, PD-ligand 1 and cytotoxic T-lymphocyte antigen 4 , has revolutionized the therapeutic strategy for patients with various types of malignant tumor, including UC (5). Pembrolizumab (Pem), a monoclonal antibody targeting PD1 , was shown to significantly prolong overall survival (OS) over chemotherapy, including paclitaxel, docetaxel or vinflunine, in patients with recurrent advanced UC that had progressed with prior platinum-based combination chemotherapy in the phase III KEYNOTE-045 trial (6). This finding was the initial demonstration of the survival benefit of any agent over chemotherapy in this category of patients, and since then, Pem has been widely accepted as a standard second-line treatment for these patients. However, findings remain insufficient regarding the comparison between Pem and chemotherapy for platinum-refractory UC patients based on data from real-world clinical practice.

Considering these findings, this study was retrospectively conducted to comparatively assess the clinical outcomes 
Table I. Characteristics of advanced urothelial cancer patients who received second-line therapy with pembrolizumab (Pem) or combined chemotherapy with gemcitabine and paclitaxel (GP).

\begin{tabular}{lccc}
\hline Variables & $\begin{array}{c}\text { Pem group } \\
(\mathrm{n}=46)\end{array}$ & $\begin{array}{c}\text { GP group } \\
(\mathrm{n}=43)\end{array}$ & $p$-Value \\
\hline $\begin{array}{l}\text { Median age (years, range) } \\
\text { Gender (\%) }\end{array}$ & $72.5(56-85)$ & $73.0(44-88)$ & 0.45 \\
$\quad$ Male & $34(73.9)$ & $28(65.1)$ & 0.49 \\
$\quad$ Female & $12(26.1)$ & $15(34.9)$ & \\
ECOG performance status (\%) & & & 0.35 \\
$\quad$ o or 1 & $35(76.1)$ & $28(65.1)$ & \\
$\geq 2$ & $11(23.9)$ & $15(34.9)$ & \\
$\begin{array}{l}\text { Site of primary tumor }(\%) \\
\quad \text { Upper urinary tract }\end{array}$ & $21(45.7)$ & $18(41.9)$ & \\
$\quad$ Lower urinary tract & $25(54.3)$ & $25(58.1)$ & \\
Liver metastasis (\%) & & & 0.83 \\
$\quad$ Positive & $6(13.0)$ & $12(27.9)$ & \\
$\quad$ Negative & $40(87.0)$ & $31(72.1)$ & \\
Surgical resection of & & & 0.83 \\
primary tumor (\%) & & & \\
$\quad$ Yes & $27(58.7)$ & $27(62.8)$ & \\
$\quad$ No & $19(41.3)$ & $16(37.2)$ & \\
First-line chemotherapy (\%) & & & 0.057 \\
$\quad \begin{array}{l}\text { Gemcitabine and cisplatin } \\
\text { Gemcitabine and carboplatin }\end{array}$ & $16(34.8)$ & $24(55.8)$ & \\
\hline
\end{tabular}

ECOG, Eastern Cooperative Oncology Group.

between Pem and combined chemotherapy with gemcitabine and paclitaxel (GP) as second-line therapy in a total of 89 advanced UC patients receiving first-line platinum-based combination chemotherapy.

\section{Patients and Methods}

Patients. This was a retrospective study performed by reviewing clinicopathological data from a total of 89 consecutive Japanese patients who were diagnosed with advanced UC and subsequently received gemcitabine plus cisplatin or carboplatin therapy followed by either Pem or GP therapy as second-line treatment in a routine clinical setting between September 2010 and March 2020. At our institution, Pem was generally applied for advanced UC patients refractory to platinum-based combination chemotherapy after the approval of this agent for previously treated UC patients in Japan. The Research Ethics Committee of our institution approved the design of this study, and the need to obtain informed consent for involvement in it from all of the included patients was waived due to its retrospective design.

Treatment. All patients included in this series had been histologically diagnosed with urothelial carcinoma of the renal pelvis, ureter, bladder and/or urethra, and initially received gemcitabine plus cisplatin or carboplatin therapy, considering several conditions, such as the symptoms and findings of a blood test, in each patient, and following the failure of first-line platinum-based combination chemotherapy, second-line therapy with either Pem or GP was introduced. In the Pem group, all patients received Pem on day 1 at a dose of $200 \mathrm{mg}$ every
Table II. Response to second-line therapy with pembrolizumab (Pem) or combined chemotherapy with gemcitabine and paclitaxel (GP) in advanced urothelial cancer patients.

\begin{tabular}{lccc}
\hline Variables & $\begin{array}{c}\text { Pem group } \\
(\mathrm{n}=46)\end{array}$ & $\begin{array}{c}\text { GP group } \\
(\mathrm{n}=43)\end{array}$ & $p$-Value \\
\hline Response $(\%)$ & & & 0.67 \\
$\quad$ Complete response & $1(2.2)$ & $1(2.3)$ & \\
$\quad$ Partial response & $11(23.9)$ & $6(14.0)$ & \\
$\quad$ Stable disease & $12(26.1)$ & $15(34.9)$ & \\
$\quad$ Progressive disease & $22(47.8)$ & $21(48.8)$ & \\
Objective response $(\%)$ & $12(26.1)$ & $7(16.3)$ & 0.31 \\
Disease control (\%) & $24(52.2)$ & $22(51.2)$ & 1 \\
\hline
\end{tabular}

3 weeks. In the GP group, G and P were administered on days 1,8 and 15 at a dose of $1,000 \mathrm{mg} / \mathrm{m}^{2}$ and on day 1 at a dose of $200 \mathrm{mg} / \mathrm{m}^{2}$, respectively, every 3 weeks. In both groups, it was possible to modify the dosage or postpone regimens, considering the degrees of treatmentassociated adverse events (AEs), and treatment was continued until disease progression or intolerable AEs occurred. Depending on the general condition and preference of each patient, GP and combined chemotherapy with methotrexate, vinblastine, doxorubicin and cisplatin were generally introduced in the Pem and GP groups, respectively, as third-line therapy.

Evaluation. In this study, clinicopathological data, including the treatment profile, were retrospectively obtained from the medical records of each patient. Prior to the initiation of second-line therapy, the Eastern Cooperative Oncology Group performance status (ECOG PS) was assessed, and radiological examinations by computed tomography (CT) of the brain, chest and abdomen and/or radionuclide bone scintigraphy were performed in all patients. As a rule, tumor measurements were performed by CT every 2 to 3 courses after the introduction of second-line therapy. In this series, disease progression was evaluated using Response Evaluation Criteria in Solid Tumors, version 1.1, and adverse events during treatment with second-line therapy were assessed using the National Cancer Institute Common Terminology Criteria for Adverse Events version 4.0.

Statistical analysis. EZR software (Saitama Medical Center, Jichi Medical University, Saitama, Japan) was employed in all statistical analyses, and $p<0.05$ was considered significant in this study. Differences in several parameters between the two groups were assessed by the unpaired $t$-test and Fisher's exact test. Progressionfree survival (PFS) was defined as the time from the start of secondline therapy to disease progression or death, while OS was defined as the time from the start of second-line therapy to death or the last follow-up. PFS and OS were calculated using the Kaplan-Meier method, and these differences were analyzed by the log-rank test. The prognostic significance of certain factors was assessed by the Cox proportional hazards regression model.

\section{Results}

Characteristics of included patients prior to second-line therapy. This study included a total of 89 patients with 
A

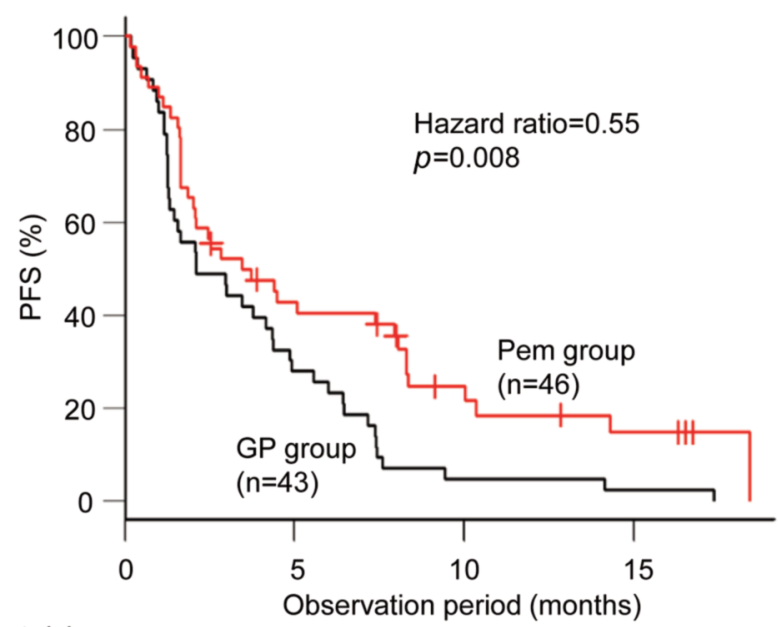

No. at risk

$\begin{array}{rrrrr}\text { Pem } & 46 & 18 & 8 & 4 \\ \text { GP } & 43 & 12 & 2 & 1\end{array}$

B

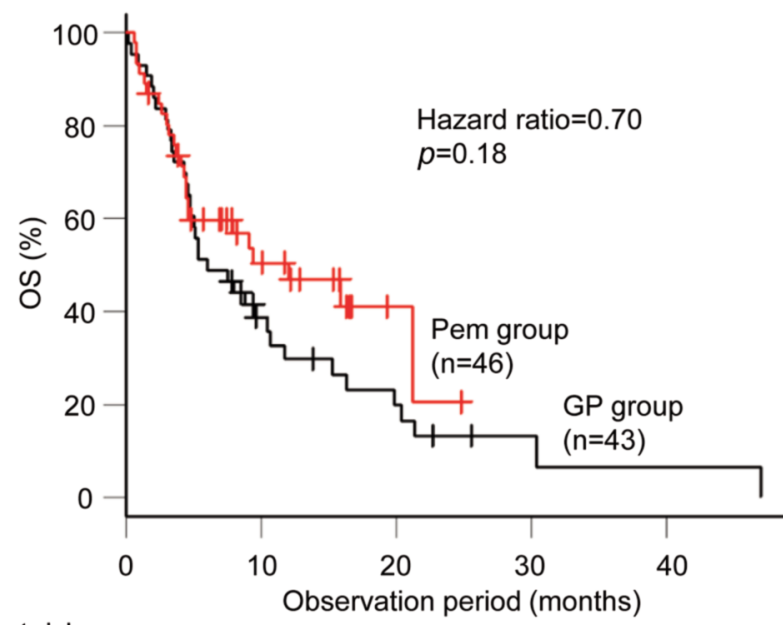

No. at risk

$\begin{array}{rrrrrr}\text { Pem } & 46 & 16 & 6 & 2 & 1 \\ \text { GP } & 43 & 13 & 2 & 0 & 0\end{array}$

Figure 1. (a) Progression-free survival (PFS) of 89 advanced urothelial cancer (UC) patients who received either pembrolizumab (Pem) or combined chemotherapy with gemcitabine and paclitaxel $(G P)$ as second-line therapy after the failure of platinum-based combination chemotherapy. (b) Overall survival (OS) of 89 advanced UC patients who received either Pem or combined chemotherapy with GP as second-line therapy after the failure of platinum-based combination chemotherapy.

advanced UC who progressed with prior platinum-based combination chemotherapy, of whom $46(51.7 \%)$ and 43 $(48.3 \%)$ received Pem and GP therapy, respectively, as second-line treatment. Table I presents a comparison of major characteristics between Pem and GP groups, and no significant differences in them were noted between these two groups.

Response to second-line therapy. Table II summarizes therapeutic outcomes of second-line therapy assessed as the best response in each group. There was no significant difference in the response pattern, objective response rate (ORR) or disease control rate between Pem and GP groups.

Prognostic outcomes after second-line therapy. In this series, disease progression occurred in 80 patients $(89.9 \%)$, including $37(80.4 \%)$ and $43(100 \%)$ in Pem and GP groups, respectively. The median PFS in the entire population, Pem and GP groups were 3.0, 3.4 and 2.1 months, respectively. As shown in Figure 1A, PFS in the Pem group was significantly longer than that in the GP group.

During the study interval, death from any cause was observed in 61 patients $(68.5 \%)$, including $24(52.2 \%)$ and $37(86.0 \%)$ in the Pem and GP groups, respectively. The median OS in the entire population, Pem and GP groups were $8.4,12.0$ and 6.0 months, respectively. As shown in Figure 1B, no significant difference in OS was noted between the two groups.
Identification of prognostic factors after second-line therapy. The impact of several factors on PFS and OS in the 89 patients were evaluated by uni- and multivariate analyses (Table III). Univariate analyses identified significant risk factors as follows: ECOG PS $\geq 2$, liver metastasis and treatment with GP for PFS, and ECOG PS $\geq 2$ and liver metastasis for OS. Furthermore, of these significant factors, ECOG PS $\geq 2$ and liver metastasis were shown to be independently associated with both PFS and OS by multivariate analyses.

AE profiles during second-line therapy. AEs associated with second-line therapy are shown in Table IV. In this series, no death due to second-line treatment-related AEs occurred. The incidence of AEs and those corresponding to grade $\geq 3$ in the GP group were significantly greater than those in the Pem group, respectively. Out of several AEs examined, peripheral neuropathy and anemia were significantly more frequently observed in the GP group than Pem group. Furthermore, the incidence of immune-related AEs (irAEs) in the Pem group was significantly greater than that in the GP group; however, there were no significant differences in that corresponding to grade $\geq 3$ between the two groups.

\section{Discussion}

Until recently, despite the lack of an established second-line therapy, several chemotherapies with insufficient evidence 
in vivo $35: 1889-1894(2021)$

Table III. Uni- and multivariate analyses of associations between various parameters with progression-free and overall survivals in advanced urothelial cancer patients who received second-line therapy with pembrolizumab (Pem) or combined chemotherapy with gemcitabine and paclitaxel (GP).

\begin{tabular}{|c|c|c|c|c|c|c|c|c|}
\hline \multirow[b]{3}{*}{ Variables } & \multicolumn{4}{|c|}{ Progression-free survival } & \multicolumn{4}{|c|}{ Overall survival } \\
\hline & \multicolumn{2}{|c|}{$\begin{array}{l}\text { Univariate } \\
\text { analysis }\end{array}$} & \multicolumn{2}{|c|}{$\begin{array}{l}\text { Multivariate } \\
\text { analysis }\end{array}$} & \multicolumn{2}{|c|}{$\begin{array}{l}\text { Univariate } \\
\text { analysis }\end{array}$} & \multicolumn{2}{|c|}{$\begin{array}{c}\text { Multivariate } \\
\text { analysis }\end{array}$} \\
\hline & $\begin{array}{l}\text { Hazard } \\
\text { ratio }\end{array}$ & $p$-Value & $\begin{array}{l}\text { Hazard } \\
\text { ratio }\end{array}$ & $p$-Value & $\begin{array}{l}\text { Hazard } \\
\text { ratio }\end{array}$ & $p$-Value & $\begin{array}{l}\text { Hazard } \\
\text { ratio }\end{array}$ & $p$-Value \\
\hline Age (years) $(>70 v s . \leq 70)$ & 1.2 & 0.5 & - & - & 0.95 & 0.83 & - & - \\
\hline Gender (male $v s$. female) & 0.89 & 0.65 & - & - & 0.77 & 0.34 & - & - \\
\hline ECOG performance status ( $\geq 2$ vs. 0 or 1 ) & 3.3 & $<0.001$ & 2.5 & $<0.001$ & 3.6 & $<0.001$ & & $<0.001$ \\
\hline $\begin{array}{l}\text { Site of primary tumor } \\
\text { (upper } v s . \text { lower urinary tract) }\end{array}$ & 1.3 & 0.29 & - & - & 1.1 & 0.64 & - & - \\
\hline Liver metastasis (positive $v s$. negative) & 3.3 & $<0.001$ & 2.2 & 0.012 & 3.6 & $<0.001$ & & 0.018 \\
\hline $\begin{array}{l}\text { Surgical resection of primary tumor } \\
\text { (yes } v s . \text { no) }\end{array}$ & 0.71 & 0.14 & - & - & 0.59 & 0.051 & - & - \\
\hline $\begin{array}{l}\text { First-line therapy } \\
(\text { GEM+CDDP } v s . \text { GEM+CBCDA) }\end{array}$ & 0.84 & 0.43 & - & - & 1 & 0.86 & - & - \\
\hline Second-line therapy (Pem vs. GP) & 0.55 & 0.0097 & 0.63 & 0.052 & 0.7 & 0.19 & - & - \\
\hline
\end{tabular}

CDDP, Cisplatin; CBCDA, carboplatin; ECOG, Eastern Cooperative Oncology Group; GEM, gemcitabine.

Table IV. Adverse events associated with second-line therapy with pembrolizumab (Pem) or combined chemotherapy with gemcitabine and paclitaxel $(G P)$ in advanced urothelial cancer patients.

\begin{tabular}{|c|c|c|c|c|c|c|}
\hline \multirow{2}{*}{$\begin{array}{l}\text { Variables } \\
\text { Events (\%) }\end{array}$} & \multicolumn{2}{|c|}{ Pem group $(n=46)$} & \multicolumn{2}{|c|}{ GP group $(n=43)$} & \multicolumn{2}{|c|}{$p$-Value } \\
\hline & Any grade & Grade $\leq 3$ & Any grade & Grade $\leq 3$ & Any grade & Grade $\leq 3$ \\
\hline Any events & $19(41.3)$ & $9(19.6)$ & $41(95.3)$ & $37(86.0)$ & $<0.001$ & $<0.001$ \\
\hline Fever & $0(0)$ & $0(0)$ & $6(14.0)$ & $1(2.3)$ & NA & NA \\
\hline Nausea & $2(4.3)$ & $1(2.2)$ & $5(11.6)$ & $0(0)$ & 0.26 & NA \\
\hline Anorexia & $2(4.3)$ & $0(0)$ & $7(16.3)$ & $1(2.3)$ & 0.083 & NA \\
\hline Alopecia & $0(0)$ & $0(0)$ & $6(14.0)$ & $0(0)$ & NA & NA \\
\hline Peripheral neuropathy & $2(4.3)$ & $0(0)$ & $23(53.5)$ & $8(18.6)$ & $<0.001$ & NA \\
\hline Anemia & $1(2.2)$ & $1(2.2)$ & $21(48.8)$ & $14(32.6)$ & $<0.001$ & $<0.001$ \\
\hline Leukopenia & $0(0)$ & $0(0)$ & $36(83.7)$ & $28(65.1)$ & NA & NA \\
\hline Neutropenia & $0(0)$ & $0(0)$ & $8(18.6)$ & $6(14.0)$ & NA & NA \\
\hline Thrombocytopenia & $0(0)$ & $0(0)$ & $22(51.2)$ & $16(37.2)$ & NA & NA \\
\hline Febrile neutropenia & $0(0)$ & $0(0)$ & $8(17.4)$ & $6(13.0)$ & NA & NA \\
\hline Immune-related adverse events & $14(30.4)$ & $6(13.0)$ & $2(4.7)$ & $1(2.3)$ & 0.0019 & 0.11 \\
\hline
\end{tabular}

NA, Not applicable.

using both single and multiple agents were performed for advanced UC patients after the failure of platinum-based first-line chemotherapy (4). In 2017, the phase III KEYNOTE-045 trial demonstrated an OS benefit with Pem compared to chemotherapy in patients with platinumrefractory advanced UC (6), and since then, Pem has been regarded as a standard second-line treatment for advanced UC patients. In Japan as well, Pem has been widely accepted for treating platinum-refractory advanced UC patients; however, to date, no study has compared efficacies between Pem and chemotherapy for such patients in real-world clinical practice. In this study we, therefore, retrospectively conducted a comprehensive evaluation of clinical outcomes in a total of 89 consecutive advanced UC patients receiving platinum-based combination chemotherapy followed by second-line tretament with either Pem or GP.

At our Institution, prior to the approval of Pem in Japan, GP therapy was generally applied for advanced UC patients 
following the failure of first-line combined chemotherapy with gemcitabine plus either cisplatin or carboplatin, since several studies reported comparatively promising findings of GP therapy for advanced UC patients (7-9). However, the efficacy of second-line GP therapy for Japanese advanced UC patients may be inferior to those reported in Western countries. Takeyama et al. treated 78 platinum-refractory advanced Japanese UC patients with combinations of G and taxanes, and reported that ORR, PFS and OS were 8.6\%, 3.5 months and 9.6 months, respectively (10). In this series, ORR, PFS and OS in the GP group were 14.0\%, 2.1 months and 6.0 months, respectively. Furthermore, a subgroup analysis of Japanese patients enrolled in the KEYNOTE-045 trial revealed that ORR, PFS and OS in the chemotherapy group were $18.2 \%, 4.9$ months and 8.3 months, respectively (11). Collectively, these findings suggest that second-line chemotherapy for Japanese patients with advanced UC has not been consistently favorable, irrespective of chemotherapy regimens.

In the KEYNOTE-045 trial, ORR, PFS and OS in the Pem group were $21.1 \%, 2.1$ months and 10.3 months, respectively (6), while in the Japanese population of this trial, ORR, PFS and OS in the Pem group were $20.0 \%, 2.0$ months and 8.3 months, respectively (11). In addition, several studies reported the Japanese real-world outcomes of second-line Pem administered in advanced UC patients (12-14). For example, Tamura et al. reported that ORR, PFS and OS were $14.6 \%, 2.5$ months and 11.9 months, respectively (12). As a rule, Pem has been introduced as a standard second-line therapy for platinum-refractory advanced UC patients immediately after the approval of this agent at our institution, and the therapeutic outcomes in the Pem group of this study were as follows: ORR, PFS and OS were $23.9 \%, 3.4$ months and 12.0 months, respectively. These findings at our institution were equivalent or slightly superior to those in previous studies. Considering the higher proportion of patients with poor PS than that in the clinical trial, the present outcomes indicate that second-line Pem could effectively control platinum-refractory advanced UC in Japanese real-world practice.

It is of interest to explore parameters associated with the prognosis in advanced UC patients receiving second-line therapy. To date, several studies identified prognostic parameters in advanced UC patients receiving Pem as second-line therapy. For example, Tamura et al. reported that poor PS, 2 or more metastatic organs and higher relative neutrophil-to-lymphocyte ratio change after 6 weeks could be used as independent predictors of poor OS in advanced UC patients treated with Pem (12), while Yasuoka et al. extracted the presence of liver metastasis, poor PS and high $\mathrm{C}$-reactive protein level as independent predictors of a poor prognosis in a similar population (13). In this series, multivariate analyses combining all patients included in this study, and ECOG PS $\geq 2$ and liver metastasis were shown to be independently associated with poor outcomes of both PFS and OS. To draw a more reliable conclusion on this topic, it is necessary to evaluate not only conventional prognostic factors but also interesting biomarkers. Another point of interest is to assess the different findings on comparing therapeutic outcomes between the present study and clinical trials. In this study, PFS, but not ORR or OS, in the Pem group was significantly superior to that in the GP group. In the KEYNOTE-045 trial, however, a lack of improved PFS with simultaneous improvement of both ORR and OS in the Pem group compared to the chemotherapy group was shown (6). Although it is difficult to clearly explain such an inconsistency, differences in characteristics of included patients and regimens in the chemotherapy group between the present study and KEYNOTE-045 trial could be involved, at least in part, in these findings.

In this series, the incidence of AEs and those corresponding to grade $\geq 3$ were significantly greater in the GP group than in the Pem group. These findings were consistent with those in the KEYNOTE-045 primary analysis population as well as in the Japanese subgroup $(6,11)$. These safety profiles of Pem could contribute to improve the quality of life (QOL) of advanced UC patients. This might be supported by results of QOL assessments in the KEYNOTE-045 trial, showing a significantly prolonged time to QOL deterioration in the Pem group compared with that in the chemotherapy group (15).

Herein, although there are several strengths, particularly valuable findings regarding comparison between Pem and chemotherapy for platinum-refractory UC patients based on real-world data, we would like to emphasize limitations of the study. Initially, this was conducted as a retrospective study with a small sample size; therefore, it is necessary to confirm the findings presented by conducting a prospective study including a much larger population of patients. Secondly, considering clinical features of ICIs, including Pem, such as a long duration of response, changes in the findings should be continuously monitored by prolonging the observation period. Finally, GP therapy may not be representative as a second-line chemotherapy for advanced UC patients; thus, impacts of other regimens should be considered, when interpreting the present outcomes.

\section{Conclusion}

The present analyses of second-line treatment for platinumrefractory advanced UC patients showed a significant PFS benefit and superior safety in the Pem group over the GP group; accordingly, Pem could be recommended as an effective and well-tolerated standard therapeutic option for Japanese advanced UC patients after the failure of first-line platinum-based chemotherapy. 


\section{Conflicts of Interest}

The Authors have no conflicts of interest to declare.

\section{Authors' Contributions}

Study conception and design, Hideaki Miyake; Acquisition of data, Ayana Takemura, Yuto Matsushita, Keita Tamura, Takayuki Sugiyama, Masao Nagata, Atsushi Otsuka; Analysis and interpretation of data, Ayana Takemura, Hideaki Miyake; Drafting of manuscript, Ayana Takemura, Hideaki Miyake.

\section{References}

1 Siegel RL, Miller KD and Jemal A: Cancer statistics, 2019. CA Cancer J Clin 69(1): 7-34, 2019. PMID: 30620402. DOI: $10.3322 /$ caac. 21551

2 Stein JP, Lieskovsky G, Cote R, Groshen S, Feng AC, Boyd S, Skinner E, Bochner B, Thangathurai D, Mikhail M, Raghavan D and Skinner DG: Radical cystectomy in the treatment of invasive bladder cancer: Long-term results in 1,054 patients. J Clin Oncol 19(3): 666-675, 2001. PMID: 11157016. DOI: 10.1200/JCO.2001.19.3.666

3 Yuasa T, Urakami S and Yonese J: Recent advances in medical therapy for metastatic urothelial cancer. Int J Clin Oncol 23(4): 599-607, 2018. PMID: 29556919. DOI: 10.1007/s10147-018$1260-0$

4 Naito R, Izumi K, Kadomoto S, Makino T, Iwamoto H, Yaegashi $\mathrm{H}$, Shigehara K, Kadono Y and Mizokami A: the efficacy of second-line chemotherapy for advanced or metastatic urothelial cancer. Anticancer Res 40(2): 1141-1146, 2020. PMID: 32014966. DOI: 10.21873/anticanres.14055

5 Keung EZ and Wargo JA: The current landscape of immune checkpoint inhibition for solid malignancies. Surg Oncol Clin N Am 28(3): 369-386, 2019. PMID: 31079794. DOI: 10.1016/ j.soc.2019.02.008

6 Bellmunt J, de Wit R, Vaughn DJ, Fradet Y, Lee JL, Fong L, Vogelzang NJ, Climent MA, Petrylak DP, Choueiri TK, Necchi A, Gerritsen W, Gurney H, Quinn DI, Culine S, Sternberg CN, Mai Y, Poehlein CH, Perini RF, Bajorin DF and KEYNOTE-045 Investigators: Pembrolizumab as second-line therapy for advanced urothelial carcinoma. N Engl J Med 376(11): 10151026, 2017. PMID: 28212060. DOI: 10.1056/NEJMoa1613683

7 Meluch AA, Greco FA, Burris HA 3rd, O’Rourke T, Ortega G, Steis RG, Morrissey LH, Johnson V and Hainsworth JD: Paclitaxel and gemcitabine chemotherapy for advanced transitional-cell carcinoma of the urothelial tract: A phase II trial of the minnie pearl cancer research network. J Clin Oncol 19(12): 3018-3024, 2001. PMID: 11408496. DOI: 10.1200/ JCO.2001.19.12.3018

8 Sternberg CN, Calabrò F, Pizzocaro G, Marini L, Schnetzer S and Sella A: Chemotherapy with an every-2-week regimen of gemcitabine and paclitaxel in patients with transitional cell carcinoma who have received prior cisplatin-based therapy. Cancer 92(12): 2993-2998, 2001. PMID: 11753976. DOI: 10.1002/1097-0142(20011215)92:12<2993::aid-cncr10108> 3.0.co;2-2
9 Kaufman DS, Carducci MA, Kuzel TM, Todd MB, Oh WK, Smith MR, Ye Z, Nicol SJ and Stadler WM: A multi-institutional phase II trial of gemcitabine plus paclitaxel in patients with locally advanced or metastatic urothelial cancer. Urol Oncol 22(5): 393-397, 2004. PMID: 15464919. DOI: 10.1016/ j.urolonc.2004.01.002

10 Takeyama Y, Kato M, Nishihara C, Yamasaki T, Iguchi T, Tamada S, Kuratsukuri K and Nakatani T: Comparison of efficacy and toxicity of second-line combination chemotherapy regimens in patients with advanced urothelial carcinoma. Int $\mathbf{J}$ Clin Oncol 23(5): 944-950, 2018. PMID: 29785621. DOI: 10.1007/s10147-018-1288-1

11 Nishiyama H, Yamamoto Y, Sassa N, Nishimura K, Fujimoto K, Fukasawa S, Yokoyama M, Enokida H, Takahashi K, Tanaka Y, Imai K, Shimamoto T, Perini R, Frenkl T, Bajorin D and Bellmunt J: Pembrolizumab versus chemotherapy in recurrent, advanced urothelial cancer in Japanese patients: A subgroup analysis of the phase 3 KEYNOTE-045 trial. Int J Clin Oncol 25(1): 165-174, 2020. PMID: 31729625. DOI: 10.1007/s10147019-01545-4

12 Tamura D, Jinnouchi N, Abe M, Ikarashi D, Matsuura T, Kato R, Maekawa S, Kato Y, Kanehira M, Takata R and Obara W: Prognostic outcomes and safety in patients treated with pembrolizumab for advanced urothelial carcinoma: Experience in real-world clinical practice. Int J Clin Oncol 25(5): 899-905, 2020. PMID: 31907720. DOI: 10.1007/s10147-019-01613-9

13 Yasuoka S, Yuasa T, Nishimura N, Ogawa M, Komai Y, Numao $\mathrm{N}$, Yamamoto S, Kondo $\mathrm{Y}$ and Yonese J: Initial experience of pembrolizumab therapy in Japanese patients with metastatic urothelial cancer. Anticancer Res 39(7): 3887-3892, 2019. PMID: 31262917. DOI: 10.21873/anticanres.13539

14 Furubayashi N, Kuroiwa K, Tokuda N, Tomoda T, Morokuma F, Hori Y, Negishi T, Inoue T, Kumagai $M$ and Nakamura M: Treating Japanese patients with pembrolizumab for platinumrefractory advanced urothelial carcinoma in real-world clinical practice. J Clin Med Res 12(5): 300-306, 2020. PMID: 32489505. DOI: 10.14740 /jocmr4162

15 Vaughn DJ, Bellmunt J, Fradet Y, Lee JL, Fong L, Vogelzang NJ, Climent MA, Petrylak DP, Choueiri TK, Necchi A, Gerritsen W, Gurney H, Quinn DI, Culine S, Sternberg CN, Mai Y, Li H, Perini RF, Bajorin DF and de Wit R: Health-related quality-oflife analysis from KEYNOTE-045: A phase III study of pembrolizumab versus chemotherapy for previously treated advanced urothelial cancer. J Clin Oncol 36(16): 1579-1587, 2018. PMID: 29590008. DOI: 10.1200/JCO.2017.76.9562 